\title{
PARTIAL RESOLUTION BY TOROIDAL BLOW-UPS
}

\author{
JÁNOS KOLLÁR
}

\begin{abstract}
We give an alternate proof of a theorem of Tevelev about improving a non-toroidal ideal sheaf by a sequence of toroidal blow-ups.
\end{abstract}

1 (Toroidal blow-up). Let $X$ be a smooth variety over a field and $\sum D_{i}$ a simple normal crossing (abbreviated as snc) divisor on $X$. A (closed) stratum of $\left(X, \sum D_{i}\right)$ is an irreducible component of an intersection $D_{i_{1}} \cap \cdots \cap D_{i_{r}}$. If $Z \subset X$ is a stratum (or a disjoint union of strata) and $\pi: B_{Z} X \rightarrow X$ the blow-up then $\left(B_{Z} X, \sum_{i} \pi_{*}^{-1} D_{i}+\sum_{j} E_{j}\right)$ is also an snc pair where the $E_{j}$ are the exceptional divisors of $\pi$. We call such blow-ups toroidal.

The following question was suggested by Keel.

Question 2. Let $\left(X, \sum D_{i}\right)$ be an snc pair over a field and $J \subset \mathcal{O}_{X}$ an ideal sheaf. How much can one improve $J$ by a sequence of toroidal blow-ups?

As a simple example, assume that $X$ is a surface. Then there are very few toroidal blow-ups: we can blow up either the curves $D_{i} \subset X$ (giving the identity map) or any of their intersection points. Thus if the cosupport of $J$ (that is, the support of $\left.\mathcal{O}_{X} / J\right)$ does not contain any strata then toroidal blow-ups have no effect on $J$. Similarly, one expects to be able to improve the singularities of $J$ along strata but not necessarily along other subvarieties. This leads to the following.

Definition 3. Let $\left(X, \Delta:=\sum D_{i}\right)$ be an snc pair over a field and $J \subset \mathcal{O}_{X}$ an ideal sheaf. We say that $J$ is toroidally resolved if its cosupport does not contain any strata.

The key step of the proof is to show that each ideal sheaf $J \subset \mathcal{O}_{X}$ has a unique toroidal hull $J \subset J^{t} \subset \mathcal{O}_{X}$ such that the toroidal resolution problem for $J$ is equivalent to the ordinary resolution problem for $J^{t}$; see Definition 17 and Proposition 20. The resolution of toroidal ideals is known over arbitrary fields by [BM06], thus we get the following answer to Question 2 ,

Theorem 4. Let $(X, \Delta)$ be an snc pair over a field (of arbitrary characteristic) and $J \subset \mathcal{O}_{X}$ an ideal sheaf. Then there is a toroidal blow-up sequence

$$
\left(X_{n}, \Delta_{n}, J_{n}\right) \rightarrow \cdots \rightarrow\left(X_{0}, \Delta_{0}, J_{0}\right):=(X, \Delta, J)
$$

such that $J_{n} \subset \mathcal{O}_{X_{n}}$ is toroidally resolved.

We state a more precise version in Theorem 10 and also explain how the ideals $J_{i}$ transform into each other, but first we apply Theorem 4 to the ideal sheaf of a divisor to get the following answer to the original question of Keel.

Tevelev pointed out that, using [DCP85, the methods of [Tev07] can easily be modified to obtain Corollary 5, see also Hac08, Uli15, Vog15 for closely related variants. In fact, Tev07] gives the stronger result that $\Pi_{*}^{-1} Y$ intersects each stratum in the expected codimension. 
Corollary 5 (Tevelev). Let $(X, \Delta)$ be an snc pair over a field and $Y \subset X$ a closed subscheme that does not contain any of the irreducible components of $\Delta$. Then there is a sequence of toroidal blow-ups $\Pi: X_{n} \rightarrow \cdots \rightarrow X_{0}:=X$ such that the birational transform $\Pi_{*}^{-1} Y$ does not contain any strata of the pair $\left(X_{n}, \Pi_{*}^{-1} \Delta+\operatorname{Ex}(\Pi)\right)$.

For another application, note that if a divisor $B$ does not contain any strata of $(X, \Delta)$ iff $(X, \Delta+\epsilon B)$ is divisorial log terminal (abbreviated as $d l t)$ for $0<\epsilon \ll 1$, cf. Kol13, 2.8]. We can thus restate the divisorial case of Corollary 5 as follows.

Corollary 6. Let $(X, \Delta)$ be an snc pair over a field and $B \subset X$ an effective divisor that does not contain any of the irreducible components of $\Delta$. Then there is a sequence of toroidal blow-ups $\Pi: X_{n} \rightarrow \cdots \rightarrow X_{0}:=X$ such that

$$
\left(X_{n}, \Pi_{*}^{-1}(\Delta+\epsilon B)+\operatorname{Ex}(\Pi)\right) \text { is dlt for } 0<\epsilon \ll 1 .
$$

The model obtained in Corollary 6 is related to the dlt modifications of $(X, \Delta+$ $\epsilon B)$ constructed in OX12] (in characteristic 0). Our models are smooth but the log canonical class need not be relatively nef. Nonetheless, this suggests that Corollary 6] might be approached using the minimal model program. A problem is that there are many different dlt modifications and most of them are singular. It is not clear to me how to guarantee smoothness using MMP.

7 (Plan of the proof of Theorem 4). Assume for simplicity that $(X, \Delta)$ is toric with torus $T$. We assume that $\Delta$ consists of all $T$-invariant divisors. We show that Theorem 4 for $J$ is essentially equivalent to a special case of resolution, usually called monomialization, of the toric ideal $J^{t}:=\sum_{\tau} \tau^{*} J$ where we sum over all $\tau \in T$. The latter is a combinatorial problem that is independent of the characteristic.

In general, $(X, \Delta)$ is locally toric in the analytic or étale topology so we need to check that the local construction of $J^{t}$ gives a global ideal sheaf $J^{t}$. This is probably well known to experts. I do not know a reference that covers everything that we need, so we go through the details.

In the precise version of Theorem 4 we further restrict the blow-ups allowed in the sequence. For this we need some definitions first.

8 (Toroidally equimultiple blow-ups). Let $X$ be a smooth variety and $J \subset \mathcal{O}_{X}$ an ideal sheaf. Let $Z \subset X$ be a smooth subvariety and $\pi: B_{Z} X \rightarrow X$ the blow-up of $Z$. Let $E \subset B_{Z} X$ denote the exceptional divisor.

Most resolution methods work with blow-up centers $Z \subset X$ such that $J$ is equimultiple along $Z$; that is, mult $z=m$ for every $z \in Z$ for some fixed $m$. We then define the birational transform of $J$ by

$$
\pi_{*}^{-1} J:=\mathcal{O}_{B_{Z} X}(m E) \cdot \pi^{*} J .
$$

(This is frequently called the 'controlled' or 'weak' transform.) This is an ideal sheaf on $B_{Z} X$. It has the pleasant property that mult $\pi_{*}^{-1} J \leq m$ for every $y \in E$.

Working toroidally, we would like $Z$ to be a stratum (or a disjoint union of strata). However, if the multiplicity of $J$ jumps at a single point that is not a stratum, then toroidal blow-ups are unlikely to change this. Thus, in a resolution procedure, the best one can hope for is that $J$ is toroidally equimultiple along $Z$, that is, mult $_{W} J=$ mult $_{Z} J$ for every stratum $W \subset Z$.

If this holds then we define the birational transform of $J$ by

$$
\pi_{*}^{-1} J:=\mathcal{O}_{B_{Z} X}(m E) \cdot \pi^{*} J
$$


As before, this is an ideal sheaf on $B_{Z} X$ and mult $_{V} \pi_{*}^{-1} J \leq m$ for every stratum $V \subset E$.

The resulting birational transform of $J$ then behaves as expected over generic points of strata $W \subset Z$ but can be rather badly behaved elsewhere. This is not a problem if we care only about generic points of strata.

Let us recall a somewhat detailed form of resolution (usually called monomialization) of ideal sheaves.

Theorem 9. Kol07, 3.68] Let $(X, E)$ be an snc pair over a field of characteristic $O$ and $J \subset \mathcal{O}_{X}$ an ideal sheaf. Then there is a blow-up sequence

$$
\left(X_{n}, J_{n}, E_{n}\right) \rightarrow \cdots \rightarrow\left(X_{0}, J_{0}, E_{0}\right):=(X, J, E)
$$

with the following properties.

(1) Each $\pi_{i}: X_{i+1} \rightarrow X_{i}$ is a blow-up with smooth center $Z_{i} \subset X_{i}$ and exceptional divisor $E^{i+1}$.

(2) $J_{i}$ is equimultiple along $Z_{i}$.

(3) $J_{i+1}=\left(\pi_{i}\right)_{*}^{-1} J_{i}$ as in (8, 1).

(4) $Z_{i}$ has normal crossings with $E_{i}$ and $E_{i+1}=\left(\pi_{i}\right)_{*}^{-1} E_{i}+E^{i+1}$.

(5) $\left(X_{n}, J_{n}, E_{n}\right)$ is resolved; that is, $J_{n}=\mathcal{O}_{X_{n}}$.

Now we can state the more precise form of Theorem 4 where we just add 'toroidal' to the formulation of Theorem 9 in a few places.

Theorem 10. Let $(X, \Delta)$ be an snc pair over a field of any characteristic and $J \subset \mathcal{O}_{X}$ an ideal sheaf. Then there is a toroidal blow-up sequence

$$
\left(X_{n}, \Delta_{n}, J_{n}\right) \rightarrow \cdots \rightarrow\left(X_{0}, \Delta_{0}, J_{0}\right):=(X, \Delta, J)
$$

with the following properties.

(1) Each $\pi_{i}: X_{i+1} \rightarrow X_{i}$ is a blow-up with smooth, toroidal center $Z_{i} \subset X_{i}$ and exceptional divisor $E_{i+1}$.

(2) $J_{i}$ is toroidally equimultiple along $Z_{i}$.

(3) $J_{i+1}=\left(\pi_{i}\right)_{*}^{-1} J_{i}$ as in (8, 2).

(4) $\Delta_{i+1}=\left(\pi_{i}\right)_{*}^{-1} \Delta_{i}+E_{i+1}$.

(5) $\left(X_{n}, \Delta_{n}, J_{n}\right)$ is toroidally resolved.

Remark 11. The role of the divisors $E$ and $\Delta$ is quite different in the two Theorems; the notation is changed to emphasize this. In Theorem $9 E$ is but an auxiliary datum which gives very mild restrictions on the blow-up centers, whereas in Theorem $4 \Delta$ gives extremely strong restrictions on the blow-up centers.

Definition 12. Let us call a blow-up sequence satisfying $(9,1-4)$ equimultiple and a blow-up sequence satisfying $[10,1-4)$ toroidally equimultiple.

Thus Theorem 9 says that, in characteristic 0 , every ideal sheaf can be resolved by an equimultiple blow-up sequence.

13 (Toroidal ideals). Let $X$ be a smooth variety and $\sum D_{i}$ an snc divisor. An ideal sheaf $I \subset \mathcal{O}_{X}$ is toroidal if $X$ is covered by open sets $U_{j}$ such that

$$
\left.I\right|_{U_{j}}=\sum_{s} \mathcal{O}_{U_{j}}\left(-\left.\sum_{i} m_{i j s} D_{i}\right|_{U_{j}}\right)
$$

for every $j$ and for suitable $m_{i j s} \in \mathbb{N}$. 
Let $Z \subset X$ be a closed stratum and $Z^{0}:=Z \backslash \cup\{W: W \subsetneq Z$ is a stratum $\}$ the corresponding open stratum. For every $z \in Z^{0} \cap U_{j}$ the $m_{i j s}$ give vectors

$$
v_{j s}:=\left(m_{i j s}: D_{i} \supset Z\right) \in \sum_{i: D_{i} \supset Z} \mathbb{N}\left[D_{i}\right]
$$

and these generate a subsemigroup

$$
M_{Z} \subset \sum_{i: D_{i} \supset Z} \mathbb{N}\left[D_{i}\right]
$$

which depends only on $Z$. For any inclusion of strata $W \subset Z$ we have the coordinate projection

$$
p_{Z, W}: \sum_{i: D_{i} \supset W} \mathbb{N}\left[D_{i}\right] \rightarrow \sum_{i: D_{i} \supset Z} \mathbb{N}\left[D_{i}\right]
$$

and the subsemigoups $M_{Z}$ satisfy the compatibility relation

$$
p_{Z, W}\left(M_{W}\right)=M_{Z} \text {. }
$$

This gives a one-to-one correspondence between toroidal ideals and collections of subsemigroups $\left\{M_{Z}\right\}$ satisfying the compatibility relations (13,5). In particular, we see that $I \mapsto I^{\text {an }}$ gives a one-to-one correspondence

$$
\text { \{toroidal ideals } \left.I \subset \mathcal{O}_{X}\right\} \leftrightarrow\left\{\text { toroidal ideals } I^{\text {an }} \subset \mathcal{O}_{X}^{\text {an }}\right\} .
$$

We claim that toroidal ideals are the only ones that can be 'canonically' associated to the stratification of an snc pair.

14 (Local stratified isomorphisms). Let $(X, \Delta)$ be an snc pair and $U_{1}, U_{2} \subset X$ open sets. An isomorphism $\phi: U_{1} \rightarrow U_{2}$ is called stratification preserving if $Z \cap U_{1}=$ $\phi^{-1}\left(Z \cap U_{2}\right)$ for every stratum $Z \subset X$. Note that our strata are the irreducible components of the intersections of the $D_{i}$, thus this is stronger than just assuming $D_{i} \cap U_{1}=\phi^{-1}\left(D_{i} \cap U_{2}\right)$ for every $D_{i}$.

We say that an ideal sheaf $I \subset \mathcal{O}_{X}$ is invariant under stratification preserving local isomorphisms if $\phi^{*}\left(\left.I\right|_{U_{2}}\right)=\left.I\right|_{U_{1}}$ holds for every such $\phi: U_{1} \rightarrow U_{2}$.

It is clear that a toroidal ideal is invariant under stratification preserving local isomorphisms and we would like to claim the converse. Unfortunately, if $X$ has no birational automorphisms then the identity map is the only stratification preserving local isomorphism. As usual, there are 3 ways to get more $U_{i}$.

Complex analytic 14.1. If $X$ is over $\mathbb{C}$, we use analytic open sets $U_{1}, U_{2} \subset X^{\text {an }}$.

Etale local 14, 2. We use étale morphisms $\tau_{i}: U \rightarrow X$ and require that $\tau_{1}^{-1}(Z)=$ $\tau_{2}^{-1}(Z)$ for every stratum $Z \subset X$.

Formal local 14.3. We use isomorphisms of complete local rings $\phi^{*}: \hat{\mathcal{O}}_{x_{2}, X} \rightarrow$ $\hat{\mathcal{O}}_{x_{1}, X}$. (If the base field is not algebraically closed we also allow residue field extensions.)

Micro local 14.4. We assume the condition on the tangent space level. That is

$$
\operatorname{Der}_{X}(-\log \Delta) \cdot I \subset I
$$

where $\operatorname{Der}_{X}(-\log \Delta)$ is the sheaf of logarithmic derivatives along $\Delta$; cf. Kol13, 3.87]. This works in characteristic 0 but not in positive characteristic. This shows that the concepts of toroidal ideal and toroidal hull (17) are related to D-balanced ideals and well-tuned ideals used in resolution. See [Kol07, Sec.3.4] for the latter notions. 
Proposition 15. Let $(X, \Delta)$ be an snc pair and $I \subset \mathcal{O}_{X}$ an ideal sheaf that is invariant under all stratification preserving local isomorphisms in any of the settings 14. 1-3). Then I is a toroidal ideal sheaf.

Proof. We explain the complex analytic case and leave the details of the other settings to the reader. By (13,6) it is enough to show that $I^{\text {an }}$ is toroidal.

Let $\mathbb{D} \subset \mathbb{C}$ denote the unit disc and $\mathbb{D}^{*}$ the punctured unit disc. We will view $\mathbb{D}^{*} \subset \mathbb{C}^{*}$ as a semigroup.

Let $Z^{0} \subset X$ be an open stratum. After reindexing the $D_{i}$, for every $z \in Z^{0}$ we can choose a neighborhood of the form $\left(0 \in \mathbb{D}^{n}\right)$ where $D_{i}=\left(x_{i}=0\right)$ for $i=1, \ldots, m$. We start with the natural $\left(\mathbb{D}^{*}\right)^{m}$ action on the first $m$ coordinates. This is a stratification preserving action.

Pick any $f=\sum_{i_{1}, \ldots, i_{m}} f_{i_{1}, \ldots, i_{m}}\left(x_{m+1}, \ldots, x_{n}\right) \cdot x_{1}^{i_{1}} \cdots x_{m}^{i_{m}} \in I^{\text {an }}$. Then

$$
\tau^{*} f=\sum_{i_{1}, \ldots, i_{m}} \chi_{i_{1}, \ldots, i_{m}} \cdot f_{i_{1}, \ldots, i_{m}}\left(x_{m+1}, \ldots, x_{n}\right) \cdot x_{1}^{i_{1}} \cdots x_{m}^{i_{m}}
$$

where $\chi_{i_{1}, \ldots, i_{m}}:\left(\mathbb{D}^{*}\right)^{m} \rightarrow \mathbb{D}^{*}$ denotes the character $\lambda_{1}^{i_{1}} \cdots \lambda_{m}^{i_{m}}$. Since the characters of a group (in this case $\left(\mathbb{C}^{*}\right)^{m}$ ) are linearly independent we see that

$$
f_{i_{1}, \ldots, i_{m}}\left(x_{m+1}, \ldots, x_{n}\right) \cdot x_{1}^{i_{1}} \cdots x_{m}^{i_{m}} \in I^{\mathrm{an}}+\left(x_{1}, \ldots, x_{m}\right)^{N}
$$

holds for every $N$. By Krull's intersection theorem this implies that

$$
f_{i_{1}, \ldots, i_{m}}\left(x_{m+1}, \ldots, x_{n}\right) \cdot x_{1}^{i_{1}} \cdots x_{m}^{i_{m}} \in I^{\text {an }} .
$$

We next use translations by $\left(c_{m+1}, \ldots, c_{m}\right)$ in the $x_{m+1}, \ldots, x_{n}$ directions to achieve that $f_{i_{1}, \ldots, i_{m}}\left(x_{m+1}+c_{m+1}, \ldots, x_{n}+c_{n}\right)$ is nonzero at $\left(x_{m+1}, \ldots, x_{n}\right)=(0, \ldots, 0)$. Thus

$$
x_{1}^{i_{1}} \cdots x_{m}^{i_{m}} \in I^{\text {an }} \quad \text { provided } \quad f_{i_{1}, \ldots, i_{m}}\left(x_{m+1}, \ldots, x_{n}\right) \not \equiv 0 .
$$

This shows that $I^{\text {an }}$ is generated by monomials in $x_{1}, \ldots, x_{m}$ hence it is toroidal.

Note that $(X, \Delta)$ is toric with torus $T$ then we need only the $T$-action in the above proof. Thus we have showed the following elementary observation.

Corollary 16. Let $(X, \Delta)$ be a smooth toric variety. Then an ideal is toric iff it is toroidal.

Now we come to the key definition, the toroidal hull of an ideal. The existence of the toroidal hull is a quite elementary observation which is at least implicit in several papers. See, for instance, the notion of the Newton polygon Kou76 and its connections with resolutions Tei04 or the D-balanced and well-tuned ideals discussed in Wło05]; see also [Kol07, Sec.3.4] for more details on the latter.

Definition-Theorem 17. Let $(X, \Delta)$ be an snc pair over a field and $J \subset \mathcal{O}_{X}$ an ideal sheaf. There is a unique, smallest toroidal ideal sheaf $J^{t} \supset J$, called the toroidal hull of $J$.

Furthermore, if $W \subset X$ is a stratum then mult $_{W} J^{t}=$ mult $_{W} J$. (A stronger version of this property is established in Lemma 19, )

Proof. As we noted in Paragraph 13, specifying $J^{t}$ is equivalent to specifying the semigroups $M_{Z}(13,3)$ and the latter can be done working in an analytic or formal neighborhood of a point $p_{0} \in Z^{0}$ of an open stratum.

Then the recipe of constructing $J^{t}$ follows from the proof of Proposition 15 
(*) Take all $f=\sum_{i_{1}, \ldots, i_{m}} f_{i_{1}, \ldots, i_{m}}\left(x_{m+1}, \ldots, x_{n}\right) x_{1}^{i_{1}} \cdots x_{m}^{i_{m}} \in J$ and add the monomial $x_{1}^{i_{1}} \cdots x_{m}^{i_{m}}$ to $J^{t}$ whenever $f_{i_{1}, \ldots, i_{m}} \not \equiv 0$.

This also shows that we have not decreased the multiplicity along $Z^{0}$ since

$$
\operatorname{mult}_{p_{0}} x_{1}^{i_{1}} \cdots x_{m}^{i_{m}}=\inf _{p \in Z^{0}} \operatorname{mult}_{p}\left(f_{i_{1}, \ldots, i_{m}} \cdot x_{1}^{i_{1}} \cdots x_{m}^{i_{m}}\right) \geq \inf _{p \in Z^{0}} \operatorname{mult}_{p} f .
$$

Corollary 18. Let $(X, \Delta)$ be an snc pair and $J \subset \mathcal{O}_{X}$ an ideal sheaf. Then $J$ is toroidally resolved iff $J^{t}=\mathcal{O}_{X}$.

The following result says that the toroidal hull commutes with toroidal blow-ups along toroidally equimultiple centers.

Lemma 19. Assume that $J$ is toroidally equimultiple along $Z$. Then

$$
\left(\pi_{*}^{-1} J\right)^{t}=\pi_{*}^{-1}\left(J^{t}\right) \text {. }
$$

Proof. The question is local on $X$ and we can even replace $X$ by its completion $\hat{X}_{x}$. Thus we may assume that $(X, \Delta)$ is toric with torus $T$ acting on $X$. Then $J^{t}=\sum_{\tau} \tau^{*} J$ where we sum of all $\tau \in T$. If $J$ is toroidally equimultiple along $Z$ with multiplicity $m$ then the same holds for every $\tau^{*} J$. Thus

$$
\pi_{*}^{-1}\left(J^{t}\right)=\mathcal{O}_{B_{Z} X}(m E) \cdot \pi^{*}\left(\sum_{\tau} \tau^{*} J\right)=\sum_{\tau}\left(\mathcal{O}_{B_{Z} X}(m E) \cdot \tau^{*} \pi^{*} J\right)=\left(\pi_{*}^{-1} J\right)^{t} .
$$

The following observations transforms the toroidal resolution problem for $J$ to the usual resolution problem for its toroidal hull. Thus the toroidal hull is a variant of the concept of tuning an ideal used in resolution; see [Kol07, 3.54].

Proposition 20. Let $(X, \Delta)$ be an snc pair over a field and $J \subset \mathcal{O}_{X}$ an ideal sheaf. There is a natural equivalence between the following sets.

(1) Toroidally equimultiple blow-up sequences for $J$.

(2) Toroidally equimultiple blow-up sequences for $J^{t}$.

(3) Equimultiple blow-up sequences for $J^{t}$.

Proof. Proposition 17 shows that $J$ is toroidally equimultiple along a stratum $Z$ iff $J^{t}$ is toroidally equimultiple along $Z$. A toroidal ideal is toroidally equimultiple along a stratum $Z$ iff it is equimultiple along $Z$. Thus in all 3 settings the blow-ups allowed at the first step are the same.

Lemma 19 guarantees that this holds for all subsequent steps by induction.

21 (Resolution of toroidal ideals). It has been long known that resolution of toric ideal sheaves is a combinatorial questions that is independent of the characteristic KKMSD73, AMRT75, Cox00, GPT02. However, we need a resolution that is obtained by an equimultiple blow-up sequence. The original toric references that I could find do not claim this and the methods do not seem to be designed for this purpose.

Resolution of toric and toroidal varieties and ideals using equimultiple blow-up sequences is proved in BM06]; see also [Bla12a, Bla12b. Note that our setting is quite a bit easier since for us all strata are smooth. (This is also the reason why we do not need to worry about imperfect fields.)

One should also note that for toroidal ideals an étale-local resolution procedure is automatically combinatorial. So, although this is not stated, the resolution method discussed in [Wło05] and Kol07, Chap.3] is combinatorial. Thus it yields the required resolution procedure for toroidal ideals over any field. 
22 (Proof of Theorem 10). By Theorem 9 (in characteristic $=0$ ) and Paragraph 21 (in characteristic $\neq 0$ ) there is an equimultiple blow-up sequence

$$
\left(X_{n}, \Delta_{n},\left(J^{t}\right)_{n}\right) \rightarrow \cdots \rightarrow\left(X_{0}, \Delta_{0},\left(J^{t}\right)_{0}\right):=\left(X, \Delta, J^{t}\right)
$$

that resolves $J^{t}$. By Proposition 20 the same sequence gives a toroidally equimultiple blow-up sequence for $J$

$$
\left(X_{n}, \Delta_{n}, J_{n}\right) \rightarrow \cdots \rightarrow\left(X_{0}, \Delta_{0}, J_{0}\right):=(X, \Delta, J) .
$$

By Lemma 19 we know that $\left(J_{n}\right)^{t}=\left(J^{t}\right)_{n}$ and the latter is $\mathcal{O}_{X_{n}}$ by assumption. Thus $J_{n}$ is toroidally resolved by Lemma 18 .

Acknowledgments. I thank S. Keel for posing the question, J. Huh and J. Tevelev for insightful comments and references. Partial financial support was provided by the NSF under grant number DMS-1362960.

\section{REFERENCES}

[AMRT75] A. Ash, D. Mumford, M. Rapoport, and Y. Tai, Smooth compactification of locally symmetric varieties, Math. Sci. Press, Brookline, Mass., 1975, Lie Groups: History, Frontiers and Applications, Vol. IV. MR 0457437 (56 \#15642)

[Bla12a] Rocío Blanco, Desingularization of binomial varieties in arbitrary characteristic. Part I. A new resolution function and their properties, Math. Nachr. 285 (2012), no. 11-12, 1316-1342. MR 2959962

[Bla12b] - Desingularization of binomial varieties in arbitrary characteristic. Part II: Combinatorial desingularization algorithm, Q. J. Math. 63 (2012), no. 4, 771-794. MR 2999984

[BM06] Edward Bierstone and Pierre D. Milman, Desingularization of toric and binomial varieties, J. Algebraic Geom. 15 (2006), no. 3, 443-486. MR 2219845

[Cox00] David A. Cox, Toric varieties and toric resolutions, Resolution of singularities (Obergurgl, 1997), Progr. Math., vol. 181, Birkhäuser, Basel, 2000, pp. 259-284. MR 1748623

[DCP85] C. De Concini and C. Procesi, Complete symmetric varieties. II. Intersection theory, Algebraic groups and related topics (Kyoto/Nagoya, 1983), Adv. Stud. Pure Math., vol. 6, North-Holland, Amsterdam, 1985, pp. 481-513. MR 803344

[GPT02] Pedro Daniel González Pérez and Bernard Teissier, Embedded resolutions of non necessarily normal affine toric varieties, C. R. Math. Acad. Sci. Paris 334 (2002), no. 5, 379-382. MR 1892938

[Hac08] Paul Hacking, The homology of tropical varieties, Collect. Math. 59 (2008), no. 3, 263-273. MR 2452307

[KKMSD73] G. Kempf, F. F. Knudsen, D. Mumford, and B. Saint-Donat, Toroidal embeddings. I, Lecture Notes in Mathematics, Vol. 339, Springer-Verlag, Berlin, 1973. MR 0335518 (49 \#299)

[Kol07] János Kollár, Lectures on resolution of singularities, Annals of Mathematics Studies, vol. 166, Princeton University Press, Princeton, NJ, 2007.

[Kol13] _ Singularities of the minimal model program, Cambridge Tracts in Mathematics, vol. 200, Cambridge University Press, Cambridge, 2013, With the collaboration of Sándor Kovács.

[Kou76] A. G. Kouchnirenko, Polyèdres de Newton et nombres de Milnor, Invent. Math. 32 (1976), no. 1, 1-31. MR MR0419433 (54 \#7454)

[OX12] Yuji Odaka and Chenyang Xu, Log-canonical models of singular pairs and its applications, Math. Res. Lett. 19 (2012), no. 2, 325-334.

[Tei04] Bernard Teissier, Monomial ideals, binomial ideals, polynomial ideals, Trends in commutative algebra, Math. Sci. Res. Inst. Publ., vol. 51, Cambridge Univ. Press, Cambridge, 2004, pp. 211-246. MR 2132653

[Tev07] Jenia Tevelev, Compactifications of subvarieties of tori, Amer. J. Math. 129 (2007), no. 4, 1087-1104. MR 2343384 
[Uli15] Martin Ulirsch, Tropical compactification in log-regular varieties, Math. Z. 280 (2015), no. 1-2, 195-210. MR 3343903

[Vog15] T. Vogiannou, Spherical Tropicalization, ArXiv e-prints (2015).

[Wło05] Jarosław Włodarczyk, Simple Hironaka resolution in characteristic zero, J. Amer. Math. Soc. 18 (2005), no. 4, 779-822 (electronic). MR 2163383 (2006f:14014)

Princeton University, Princeton NJ 08544-1000

kollar@math.princeton.edu 